\title{
Surgery-first Approach
}

\author{
${ }^{1}$ Yash A Shah, ${ }^{2}$ Shailesh V Deshmukh, ${ }^{3}$ Amol S Patil
}

\section{ABSTRACT}

Aim: This article is intended to provide an overview of the Surgery First Approach (SFA) mainly including case selection, diagnosis, treatment protocols, success rate and the potential problems encountered.

Background: The most important indication of the need for orthognathic surgery is usually the psychosocial effect resulting from the unaesthetic appearance of a dentofacial deformity. The conventional approach in treatment of such deformities till today has been an orthodontics-first approach.

Review Results: Available evidence suggest that both the SFA and the conventional approach had similar outcomes in dentofacial relationships however the relapse tendency was greater with the SFA but the total treatment duration was substantially shorter.

Conclusion: SFA treats facial aesthetics (and the skeletal malrelation) first and then occlusion, i.e. orthognathic surgery antecedes the orthodontic therapy. The concept of this technique is to utilize orthognathic surgery to eliminate the displeasing pre-surgical facial profile and promptly accomplish facial aesthetic enhancement that is usually the patient's chief complaint at the beginning of the treatment. SFA also facilitates accelerated orthodontic tooth movement thus reducing the length of post-surgical orthodontic therapy.

Clinical Significance: The optimal esthetic and functional results, significant reduction in total treatment time and high patient satisfaction led us to the postulation that SFA may represent a reasonable, cost-effective method to manage skeletal malocclusions in selected cases and that it has the potential to become a standard approach to orthognathic surgery in the future.

Keywords: Orthognathic surgery, Surgery first, Surgical orthodontics.

How to cite this article: Shah YA, Deshmukh SV, Patil AS. Surgery-first Approach. World J Dent 2017;8(4):343-350.

Source of support: Nil

Conflict of interest: None

\section{INTRODUCTION}

Clinicians are predominantly influenced by objective findings, but patients are more biased toward their

\footnotetext{
${ }^{1-3}$ Department of Orthodontics and Dentofacial Orthopedics Bharati Vidyapeeth Deemed University Dental College and Hospital, Pune, Maharashtra, India

Corresponding author: Amol S Patil, Department of Orthodontics and Dentofacial Orthopedics, Bharati Vidyapeeth Deemed University Dental College and Hospital, Pune Maharashtra, India, Phone: +917774048166, e-mail: amolp66@ yahoo.com
}

perception of their needs and values. This gives rise to the dilemma of what would one treat first, the profile or the occlusion. This dichotomy has led to the birth of the concept of SFA in the year of $1988 .^{1}$ The most important indication of the need for orthognathic surgery is usually the psychosocial effect resulting from the unesthetic appearance of a dentofacial deformity.

An increasing number of young individuals are now undergoing cosmetic surgical procedures. This is a direct consequence of the ever-increasing competition in the job scenario and the time of marriage. A couple of decades back, no one could have envisaged that orthognathic surgeries would prevail as one of the most popular cosmetic operations. Amidst this rise in orthognathic surgeries, the notion of SFA has begun to gain in popularity. ${ }^{2}$

The conventional approach until today has been an orthodontics-first approach. The conventional approach dictates that there is a phase of presurgical orthodontics preceding the orthognathic surgery. The SFA treats facial esthetics (the skeletal malrelation) first and then occlusion, i.e., orthognathic surgery antecedes orthodontic therapy. ${ }^{3}$

The disadvantages with the conventional approach are that (i) it requires two phases of orthodontic therapy, (ii) it can be quite time-consuming, (iii) there is deterioration of lip silhouette, (iv) soreness and discomfort during mastication in the course of the treatment, (v) psychosocial problems associated with the delay in responding to the patient's chief complaint concerning facial esthetics, and (vi) also because of the long-term orthodontic preparation, there may be complications such as dental caries, gingival recession, and root resorption. The minimal, or absence of, this above-mentioned presurgical orthodontic preparation required in the treatment algorithm is called SFA.

The concept of this technique, for no presurgical orthodontics or minimal presurgical orthodontics for only up to a couple of months in cases of an occlusal prematurity, is to utilize orthognathic surgery to promptly accomplish facial esthetic enhancement that is usually the patient's chief complaint at the beginning of the treatment. ${ }^{4}$

The optimal esthetic and functional results, significant reduction in total treatment time, and high patient satisfaction led to the postulation that surgery-first may represent a reasonable, cost-effective method to manage skeletal malocclusion in selected cases and that it has the potential to become a standard approach to orthognathic surgery in the future. ${ }^{5}$ 


\section{Description of the Surgery-first Concept}

The SFA defined as orthognathic surgery preceding orthodontic therapy is a paradigm shift in the combined surgical orthodontic treatment of severe jaw deformities. ${ }^{6}$

The fundamental concept intrinsic to this approach is the exclusion of the phase of presurgical orthodontic treatment and the elimination of the soft-tissue imbalance supplementing the dentofacial deformity. The prime concern in utilizing this approach is the reality that it is a complex technique that demands close collaboration of a skilled orthodontist and the oral surgeon. Estimation of the correct final occlusion is an extremely difficult task. Furthermore, the operating surgeon should be capable of aligning the skeletal components to exactly match the estimated skeletal orientations and occlusion perfectly. ${ }^{7}$

The SFA creates a semistable postsurgical occlusion in comparison with the conventional approach due to its disposition without presurgical orthodontic therapy. Hence, rigid internal fixation has been advised for preserving the occlusal stability postsurgically. ${ }^{8}$

The development of rigid internal fixation was of crucial importance in enabling the implementation of this approach. Skeletal relapse occurring due to the semistable postsurgical occlusion can be partly overcome with the help of rigid internal fixation. The mobility of the bony segments, with the conventional wire fixation, would not permit for a fixed position of the bony segments postsurgically. Thus, any attempt at tooth movement potentially resulted in change in the orientation of the skeletal components. ${ }^{7}$

The SFA is executed with bilateral sagittal split osteotomy (BSSO) with or without genioplasty and LeFort I osteotomy. ${ }^{5}$

This approach is advisable in cases that require little or no presurgical orthodontic preparation (alignment and decompensation). They are cases of (a) well-aligned or mildly crowded anterior teeth, (b) flat or mild curve of Spee, and (c) normal or mildly proclined/retroclined inclination of incisors. ${ }^{9}$

This approach can also be utilized in cases in which decompensation is needed. The question is how to position the jaw bones properly without compromising on the surgical results in such cases.

With notable presurgical planning and meticulous execution of surgery, the postsurgical orthodontic therapy also becomes straightforward and swift; patients can get the expected outcome of a near ideal facial profile and occlusion in a much lesser time period. ${ }^{9}$

The presurgical orthodontic preparation and decompensation being minimal in the SFA, there is no progressive deterioration of lip silhouette, facial esthetics, and dental function presurgically. The patient's chief complaint regarding facial esthetics is addressed and improved at the start of treatment. ${ }^{10}$ The preparation is begun with fullmouth bonding and banding, but no orthodontic archwire is to be placed before surgery. Instead, insertion of surgical archwires with surgical hooks and wafer adjustment are performed a day before surgery. Dental compensations are solved by osteotomy whenever possible. An "interim transitional occlusion" is set up postsurgically in a correct molar relationship..$^{10}$ Intermaxillary fixation is removed 4 weeks postoperatively. Orthodontics is a postsurgical adjunctive therapy to transform this interim transitional occlusion into a solid final occlusion. ${ }^{3}$

The SFA proves that it does not jeopardize the quality of the result in terms of facial esthetics and occlusion. It is not only an effective. but also efficient approach for selected cases. It enables us to achieve the desired facial esthetics and occlusion without the phase of presurgical orthodontic preparation.

The concept of SFA entails predicting and simulating dental arch alignment, decompensation of incisors, and coordination of dental arches using study models. Based on this information, decisions on the positioning of the maxilla and mandible during surgery are made for the correction of skeletal disparity. ${ }^{11}$

This concept has been widely popularized, especially in East Asian patients with mandibular prognathism.

The general guidelines for orthodontic treatment planning and surgical splint set up for mock surgery through the SFA have been enumerated. ${ }^{4,6,9,10,12}$

- The upper and lower dentitions are bonded and banded, but no archwires are placed. This is to keep the upper and lower dentitions undisturbed and solid before surgery. Orthodontic archwires are placed 2 weeks postoperatively for the alignment, whereas the osteotomized jaw bones are held steadily by the rigid fixation.

- For the model surgery, the maxilla and mandible are arranged in the planned molar relationship and with a positive overbite. The molar relationship could be arranged in a class I in nonextraction or four bicuspid extraction cases, class III in maxillary bicuspid extraction cases, and class II in mandibular bicuspid extraction cases. On establishing the molar relationship, the resultant overjet should also be determined.

- The postsurgical orthodontic treatment could begin immediately or by 6 weeks postoperatively for taking advantage of the rapid acceleratory phenomenon (RAP). The surgical splint should be removed for the tooth movement. The RAP has been experienced in patients having orthognathic surgery whose alignment and leveling and tooth movement in all three planes of space were achieved more easily and faster. The explanation for this phenomenon is the physiologic changes in the bone metabolism induced by orthognathic surgery. ${ }^{13}$ 
The specific guidelines for anteroposterior and vertical decompensation in class III cases are as follows:

- In the conventional approach, the incisors are positioned orthodontically in a proper inclination in the supporting bone to show the true extent of skeletal discrepancy before surgery, whereas in the SFA, they could be positioned surgically or orthodontically after surgery.

- The anteroposterior decompensation for proclined maxillary incisors can be achieved by extraction of the maxillary first bicuspids and anterior segmental osteotomy or by clockwise rotation of the maxilla by LeFort I osteotomy to upright the upper incisor inclination.

- The anteroposterior decompensation for moderately retroclined and crowded lower incisors could be achieved by setting up the molars in a class I relationship with an excessive incisor overjet, and then the lower incisors could be aligned postoperatively to obtain a normal overjet.

- The anteroposterior decompensation for severely retroclined and crowded lower incisors in a class III case can be achieved by extraction of the mandibular first bicuspids and anterior segmental osteotomy, setting up the molars in a class II molar relationship with an excessive incisor overjet, and then the lower incisors could be aligned postoperatively to obtain a normal overjet.

- A moderate-to-deep mandibular curve of Spee in a class III case is better leveled preoperatively or surgically by anterior segmental osteotomy to avoid the counterclockwise rotation of the mandible postoperatively. A counterclockwise rotation of the mandible improves the chin projection in class II cases with mandibular retrognathism, although it worsens the chin projection in a case of class III mandibular prognathism. To avoid the counterclockwise rotation of the mandible postoperatively, alternatively, the lower incisors could be intruded and the upper incisors at the same time could be extruded postoperatively. ${ }^{14}$

The specific guidelines for anteroposterior and vertical decompensation in class II cases are as follows:

- For a moderate-to-deep mandibular curve of Spee and proclined lower incisors in class II mandibular retrognathism, the anterior segment of the mandible could be leveled and intruded surgically through anterior segmental osteotomy so that the mandible could be advanced properly.

- Alternatively, the mandible could be surgically advanced to an edge-to-edge incisor relationship and without occlusal contact in the posterior teeth, and then postoperatively, the mandibular anterior teeth could be orthodontically intruded so that the mandible rotates upward and forward for posterior occlusal contact and a better chin projection.

The guidelines to be followed for transverse arch coordination:

Coordination of the intercanine and intermolar widths of the maxillary and mandibular dentitions in the conventional approach is done presurgically or surgically, whereas in the SFA, it is achieved either surgically or postsurgically.

- For a wide maxilla with a transverse discrepancy more than a molar width on each side, they could be coordinated surgically by a three-piece LeFort I osteotomy of the maxilla.

- For a wide maxilla with a transverse discrepancy less than a molar width on each side, they could be coordinated by postoperative orthodontic tooth movement. This can be done by setting up the buccal slope of the lingual cusps of the upper molars occluding on the lingual slope of the buccal cusps of the lower molars on both sides. The excessive buccal overjet would be solved postoperatively by the occlusal force itself and orthodontically by a constricting transpalatal arch in a short period because of RAP.

- For a narrow maxilla, surgically assisted rapid palatal expansion could be the treatment of choice.

The SFA uses surgical techniques to resolve both skeletal and dental imbalances, thus reducing the complexity of orthodontic therapy and also shortens the entire treatment time. An "interim transitional occlusion" is achieved postsurgically. Orthodontics is an adjunctive treatment postoperatively in the SFA to transform this occlusion into a solid final occlusion.

\section{Advantages over the Conventional Approach}

Usually, patients that undergo orthognathic surgical procedures for the correction of dentofacial deformities present with a chief complaint, which includes discontentment with their facial esthetics. The conventional three-phase technique demands complete incisor decompensation, which results in worsening of the facial appearance. In these patients, enhancement of facial appearance is not achieved until months later, after the intended surgery is actually executed. Undergoing surgical correction first eliminates the displeasing presurgical facial profile and addresses the chief complaint of the patient at the very start of therapy.

Furthermore, with respect to the conventional technique, it is not easy for the treating orthodontist to estimate the precise timing of the surgery. In the SFA, as the surgery is performed before the orthodontic therapy, patients have the possibility to select the timing of surgery to accommodate for the postsurgical recovery period. ${ }^{4}$ 
The SFA reduces or completely removes the phase of presurgical orthodontic therapy, provides immediate correction of the skeletal deformity by performing the surgical procedure first, and facilitates accelerated orthodontic tooth movement thus, reducing the length of postsurgical orthodontic therapy.

The advantages are discussed at length below.

Reduction in Duration of Treatment with the SFA

The reduction in duration of treatment with the SFA is credited to two major factors:

- The correction of the hard- and soft-tissue disharmony before initiating tooth movement.

- The regional acceleratory phenomenon (RAP).

The correction of the hard- and soft-tissue disharmony with the help of surgery permits the treating orthodontist to align the teeth in a normal hard- and soft-tissue surrounding, which facilitates the tooth movement. For example, in class III jaw bases, the disharmony between the maxilla and mandible precipitates in dental compensations which is nature's attempt at minimizing and masking the dentofacial deformity. This more often than not exhibits itself in the form of proclined upper anteriors and retroclined lower anteriors as an attempt to maintain tooth contact and minimize the reverse overjet. ${ }^{6}$ These compensations result in the best possible facial profile and occlusion that are biologically feasible with given skeletal imbalance. If these cases are treated with the conventional three-phase approach, the upper anteriors would have to be retroclined and the lower anteriors would have to be proclined to achieve incisor decompensation in both the dental arches and sufficient reverse overjet before finally going ahead with the surgery. Incisor decompensation places the teeth in a position that can be said to be "unnatural" for the existing skeletal malrelation and goes against all of the nature's compensatory mechanisms, which have been at work for years. The price is paid by a relatively large presurgical orthodontic phase.

Treatment times being shorter than a year even have been reported in the literature. The initial orthodontic phase in the conventional approach is the most timeconsuming stage. Bypassing this stage gives us an overall shortened treatment period of 1 to 1.5 years or even lesser. Treatment time will usually vary from orthodontist-toorthodontist depending on one's experience and standard of finishing and detailing. ${ }^{4}$

Prevention of Soft-tissue Profile Worsening in Surgeryfirst Orthodontics

Dentoalveolar decompensation performed in the first stage of the conventional approach works against all of nature's compensatory mechanisms. That is, the orthodontist attempts to accomplish a presurgical occlusion, which is opposite to what the hard- and soft-tissue components dictate. This is a major challenge in decompensating the dental arches before performing the surgery. When the surgical procedure is performed prior, the hard- and soft-tissue imbalance is corrected; thus, the alignment of teeth is done easily without the need to struggle with the biological restraints. This prompt correction of the hard- and soft-tissue disharmony is a major advantage in SFA. ${ }^{15}$

Furthermore, presurgical deterioration of soft-tissue profile due to dental decompensation is avoided, whereas prompt enhancement of facial esthetics is attained after the surgical procedure. This has an advantage from a psychological standpoint because of a reduced duration of unappealing orthodontic appliances, which is highly appreciated by the patient. ${ }^{16}$ Along with this, SFA improves symmetry, establishing the proper interjaw relation before orthodontic alignment and more efficient and effective decompensation.

Accelerated Orthodontic Tooth Movement in Surgeryfirst Orthodontics

In general, patients undergoing SFA require less time in the phase of postsurgical orthodontic therapy as compared with those being treated by the conventional approach. This is because.

- The incisor decompensation in the SFA is resolved partly by the surgical procedure, thus reducing the complexity of orthodontic treatment thereafter.

- The RAP also helps in considerably shortening the duration of treatment.

Postsurgical tooth alignment and leveling and tooth movement in all three planes of space are more readily achieved in 4 to 5 months after the surgical procedure. The more efficient postsurgical orthodontic tooth movement mentioned above is due to something known as the RAP. ${ }^{13}$

\section{Miscellaneous}

Finally, a significant number of temporomandibular disorder (TMD) symptom resolution along with excellent results with the surgical procedures of patients with mandibular prognathism using SFA have been reported. ${ }^{8}$ Relapse with surgery can be managed during the phase of postsurgical orthodontic therapy. Moreover, when sleep-disordered breathing (often at a stage of obstructive sleep apnea) is the chief reason for combined surgical orthodontic treatment, early advancement procedures help immediately increasing the dimension of the upper airway. ${ }^{17}$

As a result of these above-mentioned advantages, the popularity of the surgery-first concept in patients and their request for this approach when an orthognathic surgery procedure is foreseen are increasing steadily. 
Surgery-first Approach

\section{Virtual Three-dimensional Planning}

The main limitation of conventional surgical planning is its two-dimensional approach, a major handicap, especially in patients with facial asymmetries, when often the deformity involves all 3 dimensions.

Virtual three-dimensional (3D) planning in orthognathic surgery is especially indicated in patients with asymmetries. It allows for a detailed visualization and analysis of skeletal and dental deformities. This approach also eliminates conventional stone model surgery through computer-aided fabrication of surgical stents.

Cone beam computed tomography allows a 3D display of the craniofacial anatomy with possibilities of image segmentation, thereby, expanding the role of imaging from diagnosis to simulation of the surgical procedures and fabrication of the surgical splints in craniofacial surgery. The 3D computer-aided surgical planning techniques for craniofacial deformities allow 3D analysis and a virtual surgical plan and provide the information for fabrication of computer-manufactured surgical splints without conventional model surgery. This virtual 3D planning provides for a more thorough diagnosis and meticulous surgical treatment planning, particularly in cases of dentofacial asymmetries. ${ }^{18,19}$

\section{Treatment Protocol}

A varied number of protocols can be employed in preparing the patient for surgery despite keeping the treatment sequence intact. Furthermore, techniques utilized in performing the surgical procedure and initiating postsurgical orthodontic therapy can be varied. Most treating orthodontists will have their own personalized predilections toward certain protocols or techniques, which will have developed through their years in practice.

In general, orthodontists prefer bonding the brackets and placing the wires right before the patient goes in for surgery. A few orthodontists even prefer to bond the wire directly onto the tooth surface. Even though bonding of the wire directly onto the tooth surface is swifter, postsurgical orthodontic therapy becomes a problem as the dentition needs to be banded and bonded at this juncture. Taking into account the time required for healing postsurgery, it becomes quite a tedious job to position brackets onto the teeth while keeping the patient's discomfort at a minimum.

The wire placed before surgery differs from orthodontist to orthodontist. In contrast to the conventional approach, aligning and leveling, decompensation, and dental arch coordination are not performed in the SFA, thus making placement of an archwire difficult. Most clinicians prefer placing passive 0.019 by $0.025^{\prime \prime} / 0.017$ by 0.025 SS wires, which have been bent to adapt to
Table 1: Conventional protocol vs other protocol variations

\begin{tabular}{|c|c|}
\hline Conventional protocol & Other protocol variations \\
\hline \multirow[t]{2}{*}{$0.022 \times 0.028 "$ appliance } & $0.018 \times 0.025$ appliance \\
\hline & $\begin{array}{l}\text { Bonding of archwire directly } \\
\text { onto the tooth surface }\end{array}$ \\
\hline $\begin{array}{l}0.019 \times 0.025 " \text { SS wire adapted to } \\
\text { individual teeth before surgery }\end{array}$ & $\mathrm{NiTi}$ wire or no wire \\
\hline $\begin{array}{l}\text { Postsurgical orthodontic therapy } \\
\text { initiated } 4-6 \text { weeks after surgery } \\
\text { with NiTi or Cu NiTi }\end{array}$ & $\begin{array}{l}\text { Initiating tooth movement } \\
\text { soon after surgery }(<1 \\
\text { month) }\end{array}$ \\
\hline
\end{tabular}

each tooth as a precaution to avoid any tooth movement presurgically. While some clinicians who opt for this approach prefer using 0.014/0.016" nickel-titanium (NiTi) wires presurgically, few clinicians also prefer not placing any wires presurgically as shown in Table 1.

The reason some clinicians use NiTi wires before surgery is because it results in instant tooth movement after surgery, which is an added benefit. However, the drawback with doing this is that the opportunity to note the relapse and/or stability of the surgery before initiating orthodontic tooth movement is lost. This is because the RAP affects both the tooth movement and the alveolar bone. Therefore, many orthodontists prefer to not use $\mathrm{NiTi}$ wires or elastic force immediately postsurgery, thus preventing undesirable movements of the alveolar bases and rather wait for 4 to 6 weeks postsurgery.

The duration of maintaining the surgical splint postsurgery also varies from orthodontist to orthodontist. Some clinicians advocate using of the splint only to aid in precise positioning of the jaw segments during surgery and discontinue its use postsurgery, while in other's opinions, it should be used anywhere up to 4 to 6 weeks postsurgery. This coincides with when the postsurgical orthodontic therapy should be initiated. Furthermore, during postsurgical orthodontic therapy, vertical elastics strictly must not be used so as to allow the bite to settle with the resolution of dental compensations.

\section{Postoperative Stability and Relapse}

Skeletal stability after the SFA has been reported, in which the mean anterior relapse ranged from 1.4 to 2.2 $\mathrm{mm}$ after 10 to $13 \mathrm{~mm}$ of mandibular setback. It is suggested that horizontal stability of surgical outcomes in the mandible might be negatively influenced when the amount of surgical setback exceeds $15 \mathrm{~mm}^{12}$

Relapse tendency at $B$ point is greater in the SFA group as compared with the conventional group as shown in Table 2. A 1-mm greater initial overbite in patients with class III malocclusion contributes to $0.449 \mathrm{~mm}$ of mandibular sagittal skeletal relapse as measured at the B point. This distinct increase in the vertical dimension found after the SFA is transient and results from occlusal prematurity 
Table 2: Relapse as measured at point $B$

\begin{tabular}{lll}
\hline $\begin{array}{l}\text { Relapse at the } \\
\text { B point }\end{array}$ & $\begin{array}{l}\text { Surgery-first } \\
\text { group (\%) }\end{array}$ & $\begin{array}{l}\text { Conventional } \\
\text { group (\%) }\end{array}$ \\
\hline$>3 \mathrm{~mm}$ & 39.1 & 15.8 \\
$<1.5 \mathrm{~mm}$ & 30.4 & 57.9 \\
$>4 \mathrm{~mm}$ & 27.8 & 8.6 \\
\hline
\end{tabular}

(accentuated Curve of Spee), which subsequently diminishes during postsurgical orthodontic therapy from better intercuspation and settling of occlusion.

Most of the literature until date is restricted to a follow-up period of 12 months or less when in fact longitudinal studies are indicated. Further randomized studies with large numbers of cases are required. Previously published reports have shown successful esthetic and functional results after the SFA. Significant shortening of the total treatment period in this approach is a definitive advantage. However, the SF approach requires more invasive surgeries as a result of eliminating presurgical orthodontics. It has been shown that BSSO without presurgical orthodontic treatment is less stable than conventional orthognathic surgery for mandibular prognathism. Therefore, these factors need to be considered when orthognathic surgery with minimal or no presurgical orthodontic treatment is indicated..$^{20}$

\section{Drawbacks of the Surgery-first Technique}

It is unquestionable that the use of this concept for treatment demands an accurate diagnosis and a detailed treatment plan. The postsurgical orthodontic tooth movements have to be planned in accordance with the surgical plan, which implies that there needs to be constant communication between the operating surgeon and the treating orthodontist. The orthodontist must be highly experienced in cases involving surgical orthodontics, because postsurgery, the orthodontist is often faced with a rather complicated scenario. Hence, it is advisable that a highly experienced and skilled team take up this treatment approach.

Several difficulties and disadvantages must be considered when attempting this approach. First, the occlusion of the dental arches can never act as a template for the determination of treatment objectives. Second, the postsurgical occlusion is always an unstable one. ${ }^{9}$

Estimation of the final outcome is the toughest part with the use of this approach. With almost all surgical cases, the maxillary and mandibular study models cannot be seated in an ideal relationship due to multiple occlusal prematurities. If the prediction of the final occlusion is not accurately planned or the predicted final occlusion is not a realistic or achievable one, results would be far from satisfactory. Treatment planning for extraction cases is especially difficult when opting for the SFA. Thus, selection of case is of greatest significance. Even after the final occlusion has been precisely determined through thorough planning, the surgery must be executed to perfection as any small error will definitely compromise the outcome. Hence, the team involved should be skilled and experienced enough to know the possibilities as well as the limitations.

In contrast to the total duration of treatment, which is considerably reduced, the process of treatment planning is time-consuming. For all practical purposes, this poses as a financial issue for the clinician. Hiking the fee for treatment is the obvious answer, but this should remain reasonable to the average patient. When stainless steel wires are to be placed before the surgery, the wire must be bent to lie passively in the brackets of all teeth. This is not only challenging, but also very time-consuming for the clinician, especially if teeth are rotated and malaligned. Thus, to facilitate this presurgical bonding procedure, some clinicians bond wires directly onto the tooth surface. Although this simplifies the presurgical procedure, there remains a requirement for another appointment for bonding during the initiation of the postsurgical orthodontic therapy.

With this approach, a two-jaw surgery is preferred so as to utilize the RAP to its maximum potential. Furthermore, a two-piece or three-piece LeFort I osteotomy is needed for the correction of severe transverse discrepancies. This increase in the number of osteotomy procedures and the level of their complexity present an appreciable risk to the patient. ${ }^{6}$

Despite the evident advantages of an SFA, patients with TMD symptoms or advanced periodontal conditions are contraindicated for this approach because of the added instability to an already semistable postsurgical occlusion. Regarding the type of dentofacial anomaly, it is advisable to restrict the indications to patients that need less leveling, alignment, and decompensation. ${ }^{4}$ Therefore, cases presenting with severe crowding, class II division 2 malocclusion cases with deep overbite, etc., should be excluded. Moreover, cases indicated for surgically assisted rapid palatal expansion to correct transverse discrepancies or severe asymmetries with dental compensations in all three planes of space should also be excluded from this protocol. All of these scenarios are too complicated to predict the outcome accurately. Moreover, the dental compensations can seriously impair the postsurgical stability. ${ }^{21}$ Although the current exclusion criteria may seem rather extensive, it is expected that the indications for the SFA will gradually broaden as the experience with this approach increases and current limitations become reasonably controlled. 
Furthermore, after surgery-first correction, patients may not be enthusiastic to move on to the second orthodontic phase, leaving an outcome that would not satisfy the important clinical goal of best possible conditions for orthodontic stability.

Furthermore, because timing of surgical treatment relates to jaw growth, patients would have delay of correction until adolescent mandibular growth is considered to be complete, whereas presurgical orthodontics could be carried on during the final period of growth.

\section{The Future of the Surgery-first Concept}

Despite the multiple challenges connected with executing the surgical procedure without the presurgical orthodontic decompensation of the dental arches, the fundamentals of this concept should be integrated into the treatment planning of all surgical cases to shorten the presurgical phase. Detailed treatment planning and sequencing of the steps, i.e., prioritizing what is imperative in advance to the surgery while leaving the remaining steps for the postsurgical orthodontic phase, thus shortening the entire treatment period. By incorporating this, the patient can not only undergo the surgical procedure earlier, but the orthodontist also gets to utilize the RAP to the fullest.

The concepts involved with the use of this approach can also be adapted to meet the individual requirements of each case. The principle objective is to try and not to spend countless months in attaining the absolute ideal presurgical leveling, alignment, and decompensation. Surgery should be done as soon as the occlusion permits at least a semistable postsurgical transitional occlusion. Once more, let it be emphasized, the skill and experience of the team involved is of paramount importance while treating cases in this manner. ${ }^{6}$

The SFA offers an alternative to the orthodontics-first approach for correction of the maxillofacial deformity. The outcomes, in the way of facial esthetics, dental occlusion, and stability, are similar when using orthodontics-first and SFAs. Orthodontists should be aware of the orthognathic principles and limits in orthodontic movement, and plan postsurgical orthodontic therapy to include leveling, alignment, decompensation, and coordination of the dental arches, and occlusal intercuspation. The surgeon should be capable of performing designated osteotomy and intermaxillary fixation with occlusion bite plate on malaligned dental arches and providing the stability after skeletal repositioning. ${ }^{22}$

Undergoing an orthognathic surgical procedure before orthodontic therapy has various advantages, which not only comprises a reduced treatment period, but also increased patient satisfaction and the availability of the RAP. If the case selection is accurate and the team involved is experienced enough to estimate the final occlusion, the outcome can be very promising. However, even the minutest error in planning and predicting the final occlusion or in the surgical procedure can be quite difficult to correct. Utilizing the basics of this approach, the duration of the presurgical orthodontic therapy can at least be reduced, if not completely eliminated. In orthodontics, our priority should always be the patient's chief complaint, and the future of this approach is geared toward addressing this and reducing the overall duration of treatment without compromising the final result.

\section{REFERENCES}

1. Leelasinjaroen P, Godfrey K, Manosudprasit M, Wangsrimongkol T, Surakunprapha P, Pisek P. Surgery first orthognathic approach for skeletal Class III malocclusion corrections-a literature review. J Med Assoc Thai 2012 Nov;95(Suppl 11): S172-S180.

2. Kwon TG. Surgery-first orthognathic surgery: beyond patients' satisfaction. J Korean Assoc Oral Maxillofac Surg 2012;38:319-320.

3. Yu CC, Chen PH, Liou EJ, Huang CS, Chen YR. A surgery-first approach in surgical-orthodontic treatment of mandibular prognathism-a case report. Chang Gung Med J 2010 NovDec;33(6):699-705.

4. Liou EJ, Chen PH, Wang YC, Yu CC, Huang CS, Chen YR. Surgery-first accelerated orthognathic surgery: orthodontic guidelines and setup for model surgery. J Oral Maxillofac Surg 2011 Mar;69(3):771-780.

5. Hernández-Alfaro F, Guijarro-Martínez R, Molina-Coral A, Badía-Escriche C. "Surgery first" in bimaxillary orthognathic surgery. J Oral Maxillofac Surg 2011 Jun;69(6):e201-e207.

6. Kim, JH.; Mahdavie, NN.; Evans, CA. Guidelines for 'surgery first' orthodontic treatment. In: Bourzgui F, editor. Orthodontics-basic aspects and clinical considerations. Rijeka: InTech; 2012. Available from: http://www.intechopen.com/books/ orthodontics-basicaspects-and-clinical-considerations / orthodontic-guidelines-for-the-surgery-first-treatment-ofseveremalocclusions. [Last accessed 12/10/2016]

7. Hernández-Alfaro F, Guijarro-Martínez R, Peiró-Guijarro MA. Surgery first in orthognathic surgery: What have we learned? A comprehensive workflow based on 45 consecutive cases. J Oral Maxillofac Surg 2014 Feb;72(2):376-390.

8. Park KR, Kim SY, Park HS, Jung YS. Surgery-first approach on patients with temporomandibular joint disease by intraoral vertical ramus osteotomy. Oral Surg Oral Med Oral Pathol Oral Radiol 2013 Dec;116(6):e429-e436.

9. Yu HB, Mao LX, Wang XD, Fang B, Shen SG. The surgeryfirst approach in orthognathic surgery: a retrospective study of 50 cases. Int J Oral Maxillofac Surg 2015 Dec;44(12): 1463-1467.

10. Yang J, Lei J, Wu Y, Lu J, Ke R, Yu Q, Mu X. Skeleton first in surgical treatment of facial disharmony. J Craniofac Surg 2015 Mar;26(2):336-339.

11. Baek SH, Ahn HW, Kwon YH, Choi JY. Surgery-first approach in skeletal class III malocclusion treated with 2-jaw surgery: evaluation of surgical movement and postoperative orthodontic treatment. J Craniofac Surg 2010 Mar;21(2): 332-338. 
12. Jorge F. Anticipated benefit: a new protocol for orthognathic surgery treatment that eliminates the need for conventional orthodontic preparation. Dent Press J Orthod 2010 JanFeb;15(1):144-157.

13. Liou EJ, Chen PH, Wang YC, Yu CC, Huang CS, Chen YR. Surgery-first accelerated orthognathic surgery: postoperative rapid orthodontic tooth movement. J Oral Maxillofac Surg 2011 Mar;69(3):781-785.

14. Lee NK, Kim YK, Yun PY, Kim JW. Evaluation of post-surgical relapse after mandibular setback surgery with minimal orthodontic preparation. J Craniomaxillofac Surg 2013 Jul;41(1): 47-51.

15. Ko EW, Lin SC, Chen YR, Huang CS. Skeletal and dental variables related to the stability of orthognathic surgery in skeletal Class III malocclusion with a surgery-first approach. J Oral Maxillofac Surg 2013 May;71(5):e215-e223.

16. Villegas C, Uribe F, Sugawara J, Nanda R. Expedited correction of significant dentofacial asymmetry using a "surgery first" approach. J Clin Orthod 2010 Feb;44(2):97-103.

17. Peiró-Guijarro MA, Guijarro-Martínez R, HernándezAlfaro F. Surgery first in orthognathic surgery: a systematic review of the literature. Am J Orthod Dentofac Orthop 2016 Apr;149(4):448-462.

18. Xia JJ, Gateno J, Teichgraeber JF. New clinical protocol to evaluate craniomaxillofacial deformity and plan surgical correction. J Oral Maxillofac Surg 2009 Oct;67(10):2093-2106.

19. Hsu SS, Singhal D, Xia JJ, Gateno J, Lin CH, Huang CS, Lo LJ, Ko EW, Chen YR. Planning the surgery-first approach in surgical-orthodontic treatment with a computer aided surgical simulation (CASS) planning protocol. J Taiwan Assoc Orthod 2012;24(2)24-37.

20. Ko EW, Hsu SS, Hsieh HY, Wang YC, Huang CS, Chen YR. Comparison of progressive cephalometric changes and postsurgical stability of skeletal Class III correction with and without presurgical orthodontic treatment. J Oral Maxillofac Surg 2011 May;69(5):1469-1477.

21. Hernández-Alfaro F, Guijarro-Martínez R. On a definition of the appropriate timing for surgical intervention in orthognathic surgery. Int J Oral Maxillofac Surg 2014 Jul;43(7):846-855.

22. Huang CS, Hsu SS, Chen YR. Systematic review of the surgery-first approach in orthognathic surgery. Biomed J 2014 Jul-Aug;37(4):184-190. 